\title{
Vedolizumab: toward a personalized therapy paradigm for people with ulcerative colitis
}

This article was published in the following Dove Press journal:

Clinical and Experimental Gastroenterology

3 March 2017

Number of times this article has been viewed

\section{Robin J Dart ${ }^{1-3, *}$ \\ Mark A Samaan ${ }^{1, *}$ \\ Nick Powell ${ }^{1,4}$ \\ Peter M Irving'}

'Department of Gastroenterology, IBD Centre, St. Thomas' Hospital, 'Immunosurveillance Laboratory, The Francis Crick Institute, London, ${ }^{3}$ Division of Immunology, Infection, and Inflammatory Disease (DIIID), King's College London, ${ }^{4}$ Department of Experimental Immunobiology, Division of Transplantation and Mucosal Biology, King's College London, London, UK

*These authors contributed equally to this work

Correspondence: Peter M Irving Department of Gastroenterology, IBD Centre, First Floor College House, North Wing, St. Thomas' Hospital, Westminster Bridge Road, London SEI 7EH, UK

Tel +442071882499

Fax +442071882484

Email peter.irving@gstt.nhs.uk
Abstract: Ulcerative colitis (UC) is a chronic relapsing and remitting inflammatory bowel disease, with a characteristic leukocytic infiltration of the mucosa. Immunosuppression including anti-TNF- $\alpha$ therapy is a mainstay of treatment for many; however, systemic immunosuppression is not universally effective and is associated with potential side effects. The gut-tropic integrin $\alpha 4 \beta 7$, which is expressed on leukocytes, mediates migration from the circulation to the intestinal mucosa. Vedolizumab is a monoclonal antibody which blocks the egress of leukocytes via $\alpha 4 \beta 7$, preventing accumulation in the mucosa, and attenuating inflammation without systemic immunosuppression. Vedolizumab has been evaluated in UC in a phase III trial, demonstrating efficacy as both an induction and a maintenance agent. In this article, we review the clinical trial data and also explore the growing body of "real-world" effectiveness data, investigating response and remission rates of vedolizumab in clinical practice. In addition, we review the increasing volume of data supporting the reassuring safety profile associated with vedolizumab. Keywords: vedolizumab, ulcerative colitis, personalized therapy

\section{Introduction}

Ulcerative colitis (UC) is a chronic relapsing idiopathic inflammatory bowel disease (IBD), and while the aetio-pathogenesis is yet to be defined, its basis lies in the complex interplay between genetic, microbial and environmental factors. ${ }^{1-3}$ It is a dynamic disease; although many patients will present with initial severe symptoms which wane over time, nearly half will run a more severe disease course, with disease extension and worsening or relapsing and remitting symptoms. ${ }^{4,5}$ Identifying these "at-risk" patient groups remains one of the greatest challenges in providing tailored care at diagnosis, and while disease extent appears to be an early predictor of potential severity, there is little consensus over other risk factors for poor prognosis. ${ }^{6-8}$

UC is classically characterized by presentation with bloody diarrhea, and peak onset is in the $3 \mathrm{rd}$ and 4 th decades of life. ${ }^{9}$ However, up to $15 \%$ of UC is diagnosed in people aged over 60 years, representing a significant cohort and, given increasing polypharmacy and comorbidities in this population, targeted therapeutic approaches with the fewest off target effects are desirable. ${ }^{10}$ There is a North-South and EastWest gradient in the incidence of UC with the highest incidence being found in North America and Northern Europe, where UC has a prevalence of up to $0.5 \%$. While emerging evidence suggests that although the incidence in high prevalence areas may be plateauing, incidence in other regions is increasing, thus representing a significant and increasing problem. ${ }^{11}$ 
The impact of UC on individuals cannot be underestimated; $73 \%$ of patients have reported interference in their leisure activities, two-thirds feel UC has a negative impact on their work, and over a quarter have had to alter their work to accommodate their disease..$^{12}$ Of the emotional, physical and social factors that impact quality of life, disease activity is the major driver for the negative impact felt by patients. ${ }^{13}$ Therapeutic strategies should aim to reduce the burden of disease on individuals and restore quality of life, and there is growing evidence that treating inflammation and achieving mucosal healing not only achieves these aims but also reduces the risk of future relapse. ${ }^{14}$ It is known that persistently active disease is associated with longer term complications such as anemia, ${ }^{15}$ colonic dysfunction and an increased risk of colorectal cancer. ${ }^{16}$ In addition, persistent inflammation may result in systemic side effects such as thromboembolic complications ${ }^{17}$ and perhaps even cardiovascular morbidity. ${ }^{18}$

The estimated cost of treating IBD is $\geq £ 700 \mathrm{M}$ per year in the National Health Service (NHS), and this may be considerably higher in settings without socialized health care, such as the United States. ${ }^{19,20}$ Furthermore, costs of UC are exacerbated by significant loss of productivity, caused by the impact of disease on individuals' abilities to work, demonstrating the impact of disease to wider society. ${ }^{21}$ Although the cost of treating IBD as a whole has not changed significantly over 2 years, the availability of more therapeutic options is shifting spending away from hospitalization and surgery and toward drug costs. ${ }^{21}$

Glucocorticoids have had the biggest impact on mortality in UC; however, their side-effect profile and lack of ability to maintain remission make long-term use unacceptable. ${ }^{22} \mathrm{~A}$ significant proportion of patients will remain well controlled with maintenance 5-ASA treatment, ${ }^{23}$ although some require immunomodulation, most commonly with azathioprine, ${ }^{24}$ and there is now good evidence that anti-TNF- $\alpha$ therapy can maintain steroid-free remission. ${ }^{25,26}$ Nevertheless, both azathioprine ${ }^{27}$ and anti-TNF- $\alpha^{28}$ have limited efficacy and side-effect profiles that leave something to be desired. Furthermore, whilst recommended as treatment options in international guidelines, calcineurin inhibitors are associated with a wide range of possible side effects. ${ }^{29}$

For those who do not respond to or fail to achieve steroidfree remission with medical therapy, colectomy remains an important option; despite recent therapeutic advances, rates of colectomy for chronically active disease remain relatively unchanged. ${ }^{30,31}$ There remains, therefore, an unmet need in patients with UC.

Vedolizumab is a recent addition to the range of drugs available to treat UC. Vedolizumab targets recruitment of leukocytes to the gut by blocking the $\alpha 4 \beta 7$ integrin resulting in a reduction in inflammation and prevention of relapse in patients with UC. This review will describe the rationale for the use of vedolizumab in UC and its application in clinical practice. Furthermore, areas of further research required to tailor this therapy to individuals will be identified.

\section{Blocking immune cell recruitment - what is the rationale?}

While the initiating events in UC remain incompletely understood, the subsequent adaptive responses, as evidenced by lymphocytic accumulation in affected gut, are in part responsible for the maintenance of inflammation. ${ }^{32}$ Naïve $\mathrm{T}$ cells are primed by $\mathrm{CD} 103^{+}$dendritic cells in secondary lymphoid tissue, where they recognize their cognate antigen, undergo clonal expansion and are programmed to home to the gut in a retinoic acid-dependent mechanism. ${ }^{33,34} \mathrm{In}$ health, such processes appear designed to promote tolerance and generation of $\mathrm{T}$ regulatory cells, which help maintain intestinal homeostasis; however, when local inflammatory conditions dictate, dendritic cells prime $T$ effector responses, which when directed toward an inappropriate stimulus may lead to persistent inflammation. ${ }^{35}$

The integrin $\alpha 4 \beta 7$, targeted by vedolizumab, is a crucial receptor which directs cells to the gut. ${ }^{36}$ Expression is limited not only to circulating conventional $\mathrm{T}$ cells but also to $\mathrm{B}$ cells, myeloid, innate lymphoid and unconventional $\gamma \delta \mathrm{T}$ cells, all of which have, albeit less well understood, potential to exacerbate inappropriate immune responses..$^{37-39}$ The expression of mucosal vascular addressin cell adhesion molecule 1 (MAdCAM-1) is largely restricted to the endothelium of vessels associated with Peyer's patches and the lamina propria. MAdCAM- 1 is the major ligand for $\alpha 4 \beta 7$ permitting adhesion and egress from the circulation by $\mathrm{T}$ cells..$^{40-42}$ Its expression is upregulated in UC and, given the gut-specific expression of MAdCAM-1, a targeted approach to block the interaction between it and $\alpha 4 \beta 7$ is an attractive therapeutic strategy. ${ }^{43}$

$\alpha 4 \beta 7$ is a heterodimer; hence, the $\alpha$ and $\beta$ subunits may be shared with other integrins. ${ }^{44}$ Natalizumab is a humanized $\mathrm{mAb}$ developed against the $\alpha 4$ subunit. Although natalizumab is known to be effective in Crohn's disease (CD) ${ }^{45}$ it also blocks $\alpha 4 \beta 1$, preventing leukocyte recruitment to the central nervous system (CNS), resulting in the potential for serious side effects. ${ }^{46}$ By comparison, vedolizumab specifically targets the $\alpha 4 \beta 7$ heterodimer and is, therefore, specific for gut-tropic leukocytes. Administration of vedolizumab to healthy non-human primates results in reduced accumulation of $\beta 7^{+}$mononuclear cells in the small intestine and colon and 
a reciprocal increase in the number of circulating $\beta 7^{+}$memory T cells. ${ }^{47}$ Moreover, in keeping with gastrointestinal (GI) tract selectivity, vedolizumab was observed to bind to mononuclear cells across the GI tract, most notably in the colon, small intestine and stomach (but not the esophagus) but did not bind mononuclear cells at extra-intestinal sites, including the brain, skin and lungs. ${ }^{47}$ Therefore, vedolizumab may have a particular role to play in patients for whom systemic immunosuppression, as results from steroids, immunosuppressants and anti-TNF, is undesirable or contraindicated.

Although a major player $\alpha 4 \beta 7$ is not unique in its ability to direct leukocytes to the colon. Recent data report an overrepresentation of $\mathrm{CCR}^{+} \mathrm{T}$ cells and its ligand CCL25, in the lamina propria of inflamed mucosa of patients with UC compared with healthy controls. ${ }^{48}$ Potential roles for CCR9 have recently been reviewed in this journal. ${ }^{49}$ However, although not yet trialed in UC, clinical results of CCR9 blockade in CD have been mixed..$^{49}$ Other emerging targets such as GPR $15^{50}$ show potential for disturbing leukocyte trafficking; however, unlike with targeting of $\alpha 4 \beta 7$, these remain some distance from clinical practice. Whilst IBD has benefited from drugs developed to treat other conditions, it may be that vedolizumab could have wider implications for human health and the capacity of vedolizumab to interfere with $\mathrm{T}$ lymphocyte trafficking to the gut may have unanticipated applications. The intestine acts both as a site of infection as well as a reservoir for HIV; in an animal model, $\alpha 4 \beta 7$ blockade in concert with anti-retroviral therapy (ART) lead to sustained viral responses, and prevention of viral rebound on cessation of ART. ${ }^{51}$

\section{Pharmacology}

Vedolizumab is highly effective in terms of $\alpha 4 \beta 7$ blockade; a single dose of vedolizumab resulted in $>90 \% \alpha 4 \beta 7$ receptor occupancy at 4 weeks on circulating $\mathrm{CD}^{+} \mathrm{CD}^{2} 5 \mathrm{RO}^{+} \mathrm{T}$ cells. ${ }^{52}$ Pharmacokinetic modeling from the phase II and III trials of vedolizumab ${ }^{53}$ identified that the $\mathrm{T}_{1 / 2}$ of vedolizumab is 25.5 days (range $14.6-36$ days; 5\%-95\% percentiles). The drug distribution is best described using a two-compartment pharmacokinetic model whereby there is nonlinear elimination governed by saturable mechanisms, such as receptor occupancy, where as other non-saturable mechanisms account for linear elimination. ${ }^{53}$ The major clinical factors which negatively affect drug concentrations for vedolizumab include low serum albumin concentration and weight $>120 \mathrm{~kg} .{ }^{53}$

Much of our knowledge of the use of mAbs in UC comes from experience with anti-TNF therapy, with clinical responses influenced by clinical parameters, drug levels and anti-drug antibodies (ADAs) ${ }^{54}$ While ADAs are appreciated to be critical in anti-TNF therapy, their relevance to the pharmacokinetics of vedolizumab is still uncertain. In early trials, high rates of ADA development resulted in responses similar to placebo. ${ }^{52}$ However, when the method of drug manufacture was changed, a far lower incidence of ADAs was noted $(11 \%)^{55}$ and pooled data from the phase III studies for UC (GEMINI 1) and CD (GEMINI 2) demonstrate ADAs in only $16 \%$ of patients. ${ }^{56,57}$ The small numbers of patients with persistent ADAs, and the low titers found in the majority of patients with ADAs $(<1: 15)$, make it difficult to assess the contribution of ADAs to treatment failure. ${ }^{55}$

It is known that a combination therapy with an immunomodulator not only reduces ADA formation in anti-TNF therapy but may also alter the pharmacokinetics of these antibodies. ${ }^{58,59}$ However, there is evidence that immunomodulation has little or no effect on the pharmacokinetics of vedolizumab. ${ }^{60}$ Vedolizumab clearance is increased by $12 \%$ in the presence of ADAs, ${ }^{53}$ whereas the drug clearance for infliximab in the presence of ADAs is increased by $47.1 \%{ }^{54}$ Thus, although ADAs may have a clinically relevant effect on treatment with vedolizumab, caution should be used in extrapolating drug behaviors between different monoclonal antibodies, and given the relatively lower immunogenicity of vedolizumab, understanding the true relevance of ADAs requires further research.

\section{Efficacy and effectiveness GEMINI I}

The efficacy of vedolizumab for the induction and maintenance of remission in UC was demonstrated by GEMINI 1, a multicenter phase III randomized, placebo-controlled study of over 800 patients from 34 countries. ${ }^{56}$ Subjects had moderate-to-severe active disease that extended at least 15 $\mathrm{cm}$ from the anal verge and had had unsuccessful treatment (i.e. active disease, lack of response or unacceptable adverse events) with one or more of corticosteroids, immunosuppressive medications (i.e. thiopurine) or anti-TNF agents.

\section{Induction phase}

GEMINI 1 included two induction cohorts: a double-blind cohort $(n=374)$ randomized to placebo or vedolizumab 300 mg IV at weeks 0 and 2, and an open-label cohort $(n=521)$ used to help generate the requisite number of responders to power the maintenance phase.

GEMINI 1 achieved its primary endpoint of the induction phase, demonstrating a significant clinical response at week 6 in the treatment arm compared with placebo (47.1\% vs $25.5 \%$, $p<0.001)$. Given that there is increasing evidence that mucosal 
healing can be clinically important, ${ }^{61,62}$ this was included as a secondary end-point; defined as a Mayo endoscopic subscore 0 or 1 , mucosal healing was more frequent in the vedolizumab arm than placebo ( $40.9 \%$ vs $24.8 \%, p=0.001)$.

\section{Maintenance phase}

At the point of entering the maintenance phase of GEMINI 1, vedolizumab responders from the induction phase were rerandomized to receive placebo or vedolizumab (300 $\mathrm{mg}$ iv) administered at either four or eight weekly intervals to week 52. Non-responders to vedolizumab induction treatment all went onto receive the more intensive four weekly regimen, and patients responding to placebo induction continued on placebo maintenance. Randomization was stratified according to three factors: induction cohort, concomitant use of corticosteroids, concomitant use of immunosuppressive agents or prior anti-TNF exposure.

The primary end point of the maintenance phase was clinical remission at week 52, defined as a Mayo Clinic score of 2 or lower with no subscore higher than 1 . The results of the maintenance phase were as impressive as the induction study with significantly more vedolizumab-treated patients achieving remission than those who received placebo $(41.8 \%$ vs $15.9 \%$ for eight weekly vedolizumab vs placebo, $p<0.001$ ); rates of durable clinical response (achieved at both 6 and 52 weeks) were $56.6 \%$ versus $23.8 \%(p<0.001)$. At week 52 , mucosal healing had been achieved by $51.6 \%$ of patients on eight weekly vedolizumab compared with $19.8 \%$ on placebo $(p<0.001)$.

\section{Cochrane systematic review and meta-analysis}

A Cochrane systematic review and meta-analysis combined the data generated by GEMINI $1^{56}$ with that of three phase III studies carried out over a 12-year period. 52,55,63,64 These included dose-finding studies as well as studies primarily aimed at gaining an understanding of the safety and pharmacological properties of vedolizumab. Pooled analyses confirmed that vedolizumab is significantly superior to placebo in terms of reducing rates of patients failing to achieve remission (risk ratio $=0.86,95 \% \mathrm{CI}, 0.80-0.91$ ), failing to achieve clinical response $(\mathrm{RR}=0.82,95 \% \mathrm{CI}, 0.75-0.91)$ and failing to achieve endoscopic remission $(\mathrm{RR}=0.82,95 \% \mathrm{CI}, 0.75-0.91)$.

\section{Observational effectiveness studies}

While efficacy is defined by the achievements of clinical endpoints in the highly controlled environment of a clinical trial, there is a growing appreciation of the importance of

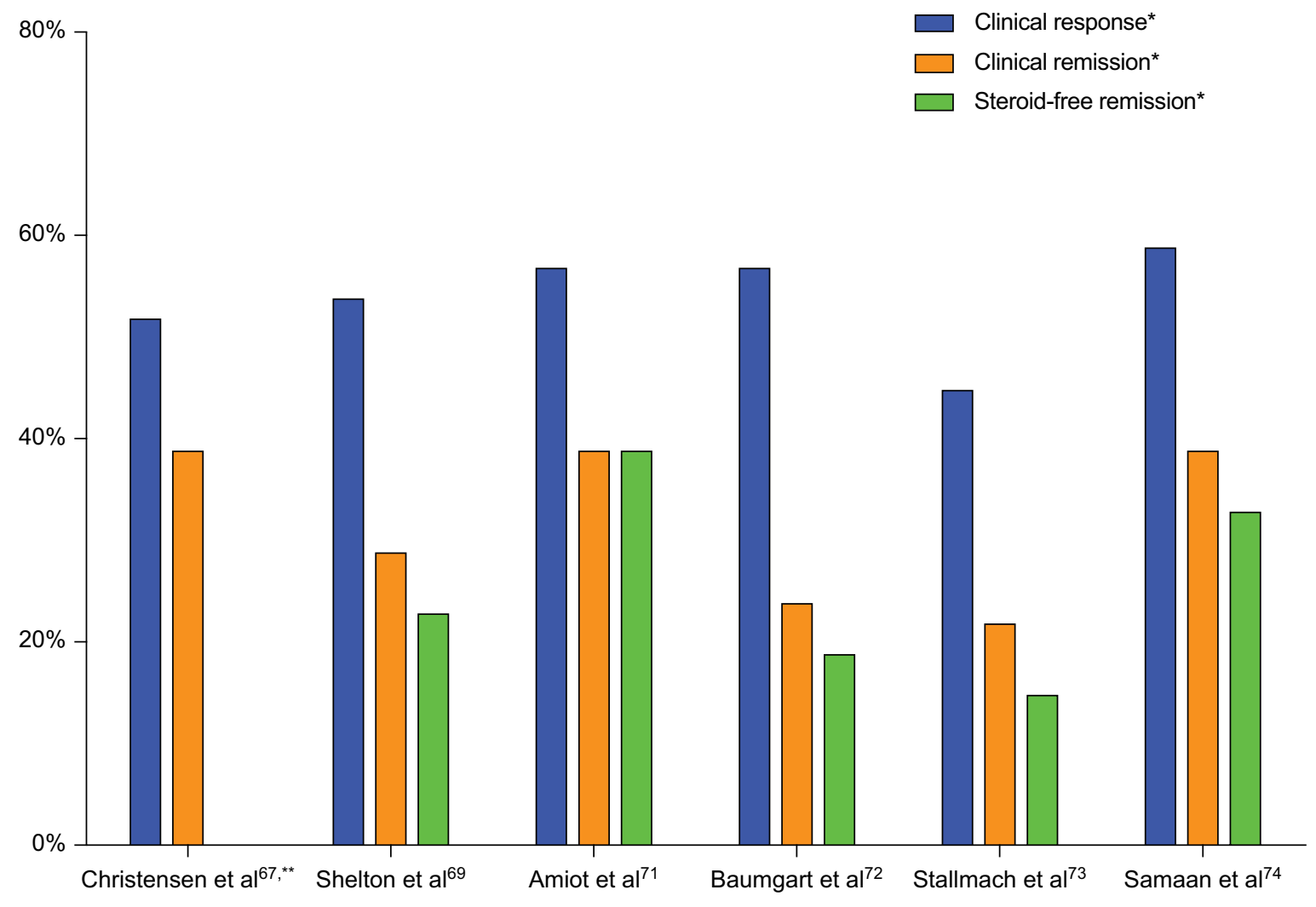

Figure I Clinical effectiveness studies of vedolizumab in ulcerative colitis. Notes: *At week 14, **data not available for steroid-free remission. 
observational, real-world effectiveness studies of drugs used in clinical practice. ${ }^{65,66}$ With this in mind, several groups in the USA and Europe have published their "real-world" experience of using vedolizumab to compliment the findings of the GEMINI trials (Figure 1).

\section{United States experience}

The University of Chicago reported experience of treating 110 IBD patients (78 CD and $32 \mathrm{UC}) .{ }^{67}$ Using the Simple Clinical Colitis Activity Index ${ }^{68}$ (SCCAI), response was defined as a drop in 3 points, and remission defined as an $\mathrm{SCCAI}<3$. Although a relatively refractory group of patients (the majority of UC patients had failed a single anti-TNF agent $[47 \%]$ with a significant proportion having failed multiple anti-TNF agents [19\%]), on analyzing patients with active disease $(n=23)$, they reported week 14 clinical response of $52 \%$ and remission rates $39 \%$. Overall, there was a mean SCCAI fall from 4.5 at baseline to 2.9 at week 14 with a further drop to 1.7 being seen at week 30 .

Another study included 172 patients (107 CD, 59 UC and 6 IBD-unclassified), prospectively assessed at two large academic centers in Boston. ${ }^{69}$ Importantly, they identified the fact that only $36 \%$ of their included patients would have met the entry criteria used in the GEMINI trials, stating reasons such as having an ileostomy/colostomy or ileoanal pouch as exclusion criterion. This finding alone further underscores the importance of "real-world" observational studies. Using the same methodology as the Chicago group, they reported clinical response and remission rates of $54 \%$ and $29 \%$, respectively, among the 58 patients with data available to week 14. In addition, data regarding corticosteroid use at week 14 demonstrated a corticosteroid-free remission rate of $23 \%$.

A group from the Washington University IBD Centre added to the growing understanding of vedolizumab's clinical effectiveness describing its use in 102 of their patients. ${ }^{70}$ Their observational study included both prospective and retrospective cohorts and also provides some endoscopic data. Of the UC patients in the prospective cohort who completed 14 weeks of treatment, $55 \%$ achieved remission (partial Mayo score $\leq 2$ with no subscore higher than 1). These patients also demonstrated significant improvements in partial Mayo scores at weeks $6(-2.6, p=0.002)$ and $14(-2.9, p=0.0002)$. In addition, an endoscopic response was also demonstrated; in the 29 patients with a Mayo endoscopic subscore of 2 (moderate) or 3 (severe) at baseline, a mucosal healing rate of $69 \%$ was observed (where mucosal healing was defined as a Mayo endoscopic subscore of 0 or 1 ).

\section{European experience}

In February 2016, the French GETAID group (Groupe d'Etude Therapeutique des Affections Inflammatoires du tube Digestif) published the results of their multicenter study of 121 vedolizumab-treated UC patients via a compassionate early access program. ${ }^{71}$ Again, this cohort included a high proportion of patients with refractory disease, $98 \%$ having been exposed to one anti-TNF agent and 69\% having used two. Using the same end-point definitions as GEMINI 1, at week 14 , they reported a $39 \%$ remission rate $36 \%$ steroidfree) and a 57\% response rate. In addition, they explored predictors of clinical effectiveness and found that a clinical response at week $6(p<0.001)$, a Mayo Clinic score $>9$ $(p=0.002)$, a C-reactive protein level $>20 \mathrm{mg} / \mathrm{L}(p=0.005)$, a leukocyte count $>8500 \times 10^{-9} / \mathrm{L}(p=0.02)$ and male gender $(p=0.09)$ were predictive of steroid-free remission at week 14 .

Following-on from the GETAID experience, and using the same methodology, the German vedolizumab consortium published a nationwide cohort study, in which data were prospectively collected on 115 UC patients. ${ }^{72}$ They demonstrated similar effectiveness to that previously described, namely, week 14 response and remission rates of $57 \%$ and $24 \%$, respectively. The German group identified a different set of predictors of clinical effectiveness to those found by GETAID. Their data suggested that active or previous smoking $(p=0.044 / 0.028)$ and a lack of prior anti-TNF exposure $(p=0.023)$ were associated with clinical remission at week 14. This data was subsequently complemented by a longer-term study that included a sample of patients from the original cohort as well as additionally recruited individuals. ${ }^{73}$ As well as week 14 outcomes, which were broadly similar to previously discussed studies (Figure 1); one-year outcomes amongst 60 UC patients were reported, demonstrating clinical remission and steroid-free clinical remission rates of $25 \%$ and $22 \%$, respectively. Of note, non-response at week 14 indicated a low likelihood of clinical remission at week 54 .

Combined, retrospective observational data from our own center (Guy's and St Thomas Hospital) and King's College Hospital, using SCCAI to measure disease activity, demonstrated similar effectiveness to the studies described above. ${ }^{74}$ Clinical disease activity among 23 UC patients was seen to fall from a median SCCAI of 6 at baseline to 4 at weeks $14(p=0.005)$ and 2 for the 10 patients followed to week $30(p=0.023)$, resulting in a week 14 remission rate of $39 \%$ (33\% corticosteroid free) and response rate of $55 \%$. By week 30, remission was seen in 50\% of patients (all of whom were corticosteroid free) and response in a further $10 \%$. In a combined analysis with CD patients, a significant 
fall in median fecal calprotectin between baseline and week 14 was also observed (1076 $\mu \mathrm{g} / \mathrm{g}$ vs $476 \mu \mathrm{g} / \mathrm{g}$ [ $p=0.010])$.

\section{Comparative studies}

Currently, the results of direct head-to-head trials of biologic therapies in UC are unavailable although this area is now being addressed. For example, a head-to-head trial of etrolizumab (a selective leukocyte adhesion molecule inhibitor with a mechanism similar to vedolizumab) and infliximab in anti-TNF-naïve patients is currently underway. ${ }^{75}$

In the absence of such data, Danese et al performed a network meta-analysis of randomized controlled trials (RCTs) assessing biological agents as induction or maintenance therapy for moderately to severely active UC. ${ }^{76}$ Using Bayesian statistical methods, eight RCTs of infliximab, adalimumab, golimumab and vedolizumab were included in the analysis. Methodological differences in trial design compounded the comparison of these drugs as maintenance agents; however, the performance of vedolizumab and antiTNF therapy as induction agents in anti-TNF-naïve patients was broadly similar.

However, as the authors acknowledge, the value of this type of analysis is intrinsically limited, and the need for head-to-head comparisons between therapeutic agents as well as real-world studies addressing both clinical and costeffectiveness remains.

\section{Relevant case series}

Large clinical trials do not address the utility of vedolizumab in special circumstances, such as pouchitis after restorative proctocolectomy and ileal-pouch anal anastomosis. A subgroup from the Boston cohort describes the effects of vedolizumab in eight patients with pouch inflammation (due either to refractory pouchitis or CD of the pouch). ${ }^{69} \mathrm{Six}$ (75\%) patients responded (reduction in modified pouchitis activity index (mPDAI) by 2 or more) and one (13\%) achieved remission (mPDAI of 4 or less).

Autoimmune liver disease (AILD) is associated with UC, in the form of primary or autoimmune sclerosing cholangitis (PSC and AISC). Furthermore, AILD is associated with aberrant expression of MADCAM-1 on liver endothelium, ${ }^{77}$ providing a rationale for the use of vedolizumab in this setting as well as in the treatment of PSC. ${ }^{78}$

We evaluated vedolizumab treatment in a cohort of 10 patients with UC and AILD, half of whom had had previous liver transplants. Clinical response (SCCAI reduction of 3 or more) at week 14 was seen in 4/10 (40\%) and remission (SCCAI <3) in $1 / 10(10 \%)$. Importantly, in a group of patients who were already on immunosuppression, there was no signal that vedolizumab treatment resulted in an increase in infectious complications, ${ }^{79}$ although the cohort is clearly small and has a relatively short follow-up.

\section{Safety and tolerability Accumulated safety data}

As described above, natalizumab is an effective treatment for $\mathrm{CD}$ and, as a result of its ability to prevent leukocyte trafficking into the CNS, multiple sclerosis. Unfortunately, in a small proportion of patients, this resulted in the occurrence of progressive multifocal leukoencephalopathy (PML) caused by reactivation of JC virus with devastating neurological degeneration which can be fatal. This led to intense scrutiny during the development of vedolizumab, and a comprehensive safety-monitoring program, RAMP (Risk Assessment and Minimization for PML), which involved detailed screening and monitoring for clinical signs of neurological dysfunction.

Using the frequency of PML cases associated with natalizumab, it can be extrapolated that the expected incidence of PML in vedolizumab-treated patients would be $\sim 2.1$ cases per 1000 patients; ${ }^{80}$ however, no cases were reported in the 2884 patients with IBD screened through RAMP nor have any been described in clinical practice to date. Experimental data demonstrate that unlike natalizumab, vedolizumab does not prevent development of inflammatory lesions in animal models of multiple sclerosis ${ }^{81}$ and does not impact leukocyte recruitment to the CNS. ${ }^{82}$ Hence, when combining experimental and observational patient data, any association between vedolizumab and PML is highly unlikely.

The systemic immunosuppressive effects of vedolizumab have also been examined; in 2014, Wyant et al ran an elegantly designed study using oral (cholera) and parenteral (hepatitis B) vaccination in patients who received either vedolizumab or placebo. ${ }^{83}$ As might be expected, vedolizumab was observed to attenuate mucosal immune responses (judged by IgA titers) to the orally administered vaccination but had no effect on immune responses to parenteral vaccination, supporting the notion that this tissue selective therapy has reduced systemic effects.

The largest study of vedolizumab's long-term safety was published in early 2016 and comprised over 2800 patients, treated for up to 5 years among six clinical studies (two phase II and four phase III studies). ${ }^{84}$ These findings were reassuring with the risk of serious opportunistic infections (4.3/100 person-years), and the rate of malignancy $(0.1 / 100$ person-years) consistent with that observed in IBD patients 
in general. Enteric infections were uncommon among vedolizumab-treated patients and if present were usually characterized by non-specific gastroenteritis. Other enteric infections such as abscesses of the GI tract or perianal region were less frequent in patients treated with vedolizumab than those receiving placebo.

\section{Real-world cautions}

In the observational study carried out by Vivio et al, six of 21 patients $(29 \%)$ in the prospective cohort and two of 30 $(7 \%)$ in the retrospective cohort experienced adverse events. ${ }^{70}$ Six of these adverse events related to worsening of UC disease activity resulting in the need for surgery, while the other two were a self-limiting febrile illness and an episode of conjunctivitis. The Chicago group reported adverse events in $41 \%$ of patients, although the majority of these were relatively minor or related to worsening disease activity. However, there was a note of caution raised by four episodes of Clostridium difficile infection among the 110 treated patients (4\%) albeit in an area in which $C$. difficile prevalence is high.

\section{Patient perspectives}

Health-related quality of life (HRQL) was included as an exploratory endpoint in GEMINI 1 and was assessed using the IBDQ at weeks 6, 30 and $52 .{ }^{56}$ The IBDQ is well validated and widely used, with higher scores (scores range $0-224$ ) indicating a better quality of life..$^{85}$ The increase in IBDQ between baseline and week 6 was significantly greater among vedolizumab-treated patients than in those on placebo $(p<0.0001)$. Furthermore, during the maintenance phase of treatment several measures of HRQL, including IBDQ, improved compared with patients treated with placebo, particularly in the groups with lower baseline disease activity or who were naïve to anti-TNF therapy. ${ }^{86}$

From the patient perspective, tolerability is crucial. This was evaluated indirectly in the observational cohort from Washington, who found that vedolizumab was generally well tolerated and that $90 \%$ of UC patients continued treatment to week 14 and $84 \%$ to week 52. In addition, vedolizumab infusion reactions are relatively rare, the majority being mild or moderate and not necessitating treatment withdrawal. Long-term safety data suggest that they occur in $<5 \%$ of patients and that the most frequently reported symptoms are nausea and headache. In the vast majority of cases (>99\%), the infusions were neither interrupted nor incomplete despite these reactions..$^{84}$

Vivio et al offered some insights from clinical practice; studying 102 IBD patients (45 UC, $57 \mathrm{CD}$ ) they found that the rates of treatment persistence at 52 weeks were $84 \%$ and $74 \%$ in $\mathrm{UC}$ and $\mathrm{CD}$, respectively. Thus suggesting that the drug is generally well tolerated by most patients. ${ }^{70}$ Furthermore, experience from infliximab demonstrates that non-adherence to eight weekly infusion treatment schedules in IBD is generally low ${ }^{87}$ and may be lower than is seen with similar drugs administered subcutaneously. ${ }^{88}$

\section{Personalizing therapy}

Identifying individuals who will benefit most from specific therapies is challenging but of paramount importance. Effective application of highly targeted, tissue-specific therapies requires a greater focus on understanding mechanisms of disease and may facilitate development of biomarkers of response. For example, etrolizumab, another monoclonal antibody targeting immune cell trafficking, binds to $\beta 7$ and, therefore, blocks both $\alpha 4 \beta 7$ and the epithelial homing integrin $\alpha_{E} \beta 7$. Higher baseline gene expression levels of ITGAE (the gene encoding $\alpha_{\mathrm{E}}$-integrin) and GZMA (granzyme A) were associated with response to etrolizumab in a recent phase II trial. ${ }^{89}$ Although this type of data is not yet available for vedolizumab, by understanding the pathogenesis of $\mathrm{UC}$, it is to be hoped that assays can be developed to target treatments to the patients for whom the benefit is likely to be greatest.

\section{Conclusion}

\section{Positioning of vedolizumab in UC treatment algorithms}

Ideally, treatment strategy in UC is tailored to individuals. Given our relative inability to define disease prognosis at onset, several factors must be taken into account when choosing a treatment regimen, as recommended by the European Crohn's and Colitis Organisation consensus statements. ${ }^{29}$ Disease activity and distribution, frequency and severity of relapses, and previous exposure to treatments must be considered. Furthermore, the relative risks and benefits of different treatment strategies need to be considered. The choice of mechanism for first-line biologic treatment in UC is currently of great interest. However, in view of the fact that head-to-head data are lacking, the choice of therapy comes down to a combination of the clinical situation and patient choice. The pros and cons in an individual setting are probably best addressed by a multidisciplinary specialist IBD team. We, therefore, discuss all relevant aspects of cases where a biologic is being considered as part of a weekly virtual biologics and immunosuppressives clinic (VBIC). ${ }^{90}$ This includes IBD-specific factors such as the predominance of extra-intestinal manifestations or perianal disease (where 
anti-TNF may be preferred) as well as medical comorbidities such as predisposition to or history of malignancy or infection (where vedolizumab may be preferred). In addition, practical factors such as patient preference for route of administration and the management of pressures on infusion suite capacity may need to be considered.

Vedolizumab is an effective therapy for UC and the focus must now be on defining its role in an expanding therapeutic market and exploring biomarkers of response to facilitate a truly personalized approach fit for the 21 st century.

\section{Disclosure}

Mark A Samaan has received advisory board fees from Hospira and lecture fees from Hospira, Takeda, and MSD. Nick Powell has received advisory board fees from Abbvie, Allergan, Debiopharm International, Ferring, and Vifor Pharma and lecture fees from Allergan and Falk Pharma. Peter M Irving has received lecture fees from Abbvie, Warner Chilcott, Ferring, Falk Pharma, Takeda, MSD, Janssen, Shire, and Tillotts; financial support for research from MSD and Takeda and Janssen; and advisory board fees from Abbvie, Warner Chilcott, Takeda, MSD, Vifor Pharma, Pharmacosmos, Topivert, Genentech, Hospira, Samsung Bioepis, Janssen, and VH2. Robin J Dart reports no conflicts of interest in this work.

\section{References}

1. Jostins L, Ripke S, Weersma RK, et al. Host-microbe interactions have shaped the genetic architecture of inflammatory bowel disease. Nature. 2012;491(7422):119-124.

2. Kostic AD, Xavier RJ, Gevers D. The microbiome in inflammatory bowel disease: current status and the future ahead. Gastroenterology. 2014;146(6):1489-1499.

3. Rogler G, Vavricka S. Exposome in IBD: recent insights in environmental factors that influence the onset and course of IBD. Inflammatory Bowel Diseases. 2015;21(2):400-408.

4. Solberg IC, Lygren I, Jahnsen J, et al. Clinical course during the first 10 years of ulcerative colitis: results from a population-based inception cohort (IBSEN Study). Scand J Gastroenterol. 2009;44(4):431-440.

5. Langholz E, Munkholm P, Davidsen M, Nielsen OH, Binder V. Changes in extent of ulcerative colitis: a study on the course and prognostic factors. Scand J Gastroenterol. 1996;31(3):260-266.

6. Waterman M, Knight J, Dinani A, et al. Predictors of Outcome in Ulcerative Colitis. Inflammatory Bowel Diseases. 2015;21(9):2097-2105.

7. Kiss LS, Lakatos PL. Natural history of ulcerative colitis: current knowledge. Current Drug Targets. 2011;12(10):1390-1395.

8. Satsangi J, Silverberg MS, Vermeire S, Colombel JF. The Montreal classification of inflammatory bowel disease: controversies, consensus, and implications. Gut. 2006;55(6):749-753.

9. Loftus EV, Jr. Clinical epidemiology of inflammatory bowel disease: Incidence, prevalence, and environmental influences. Gastroenterology. 2004;126(6):1504-1517.

10. Loftus E, Silverstein M, Sandborn W, Tremaine W, Harmsen W, Zinsmeister A. Ulcerative colitis in Olmsted County, Minnesota, 19401993: incidence, prevalence, and survival. Gut. 2000;46(3):336-343.

11. Molodecky NA, Soon IS, Rabi DM, et al. Increasing incidence and prevalence of the inflammatory bowel diseases with time, based on systematic review. Gastroenterology. 2012;142(1):46-54 e42; quiz e30.
12. Ghosh S, Mitchell R. Impact of inflammatory bowel disease on quality of life: Results of the European Federation of Crohn's and Ulcerative Colitis Associations (EFCCA) patient survey. Journal of Crohn's \& Colitis. 2007;1(1):10-20.

13. Irvine EJ. Quality of life of patients with ulcerative colitis: past, present, and future. Inflammatory Bowel Diseases. 2008;14(4):554-565.

14. Froslie KF, Jahnsen J, Moum BA, Vatn MH, Group I. Mucosal healing in inflammatory bowel disease: results from a Norwegian populationbased cohort. Gastroenterology. 2007;133(2):412-422.

15. Goodhand JR, Kamperidis N, Rao A, et al. Prevalence and management of anemia in children, adolescents, and adults with inflammatory bowel disease. Inflammatory Bowel Diseases. 2012;18(3):513-519.

16. Rutter M, Saunders B, Wilkinson K, et al. Severity of inflammation is a risk factor for colorectal neoplasia in ulcerative colitis. Gastroenterology. 2004;126(2):451-459.

17. Irving PM, Pasi KJ, Rampton DS. Thrombosis and Inflammatory Bowel Disease. Clinical Gastroenterology and Hepatology. 2005;3(7): 617-628.

18. Andersen NN, Jess T. Risk of cardiovascular disease in inflammatory bowel disease. World J Gastrointest Pathophysiol. 2014;5(3):359-365.

19. Bassi A, Dodd S, Williamson P, Bodger K. Cost of illness of inflammatory bowel disease in the UK: a single centre retrospective study. Gut. 2004;53(10):1471-1478.

20. Kappelman MD, Rifas-Shiman SL, Porter CQ, et al. Direct health care costs of Crohn's disease and ulcerative colitis in US children and adults. Gastroenterology. 2008;135(6):1907-1913.

21. van der Valk ME, Mangen MJ, Severs M, et al. Evolution of costs of inflammatory bowel disease over two years of follow-up. PLoS One. 2016;11(4):e0142481.

22. Edwards FC, Truelove SC. The course and prognosis of ulcerative colitis. Gut. 1963;4:299-315.

23. Sutherland L, Macdonald JK. Oral 5-aminosalicylic acid for maintenance of remission in ulcerative colitis. The Cochrane Database of Systematic Reviews. 2006(2):Cd000544.

24. Ardizzone S, Maconi G, Russo A, Imbesi V, Colombo E, Bianchi Porro G. Randomised controlled trial of azathioprine and 5-aminosalicylic acid for treatment of steroid dependent ulcerative colitis. Gut. 2006;55(1):47-53.

25. Rutgeerts P, Sandborn WJ, Feagan BG, et al. Infliximab for induction and maintenance therapy for ulcerative colitis. The New England Journal of Medicine. 2005;353(23):2462-2476.

26. Sandborn WJ, van Assche G, Reinisch W, et al. Adalimumab induces and maintains clinical remission in patients with moderate-to-severe ulcerative colitis. Gastroenterology. 2012;142(2):257-265 e251-253.

27. Louis E, Irving P, Beaugerie L. Use of azathioprine in IBD: modern aspects of an old drug. Gut. 2014;63(11):1695-1699.

28. Ford AC, Peyrin-Biroulet L. Opportunistic infections with anti-tumor necrosis factor-alpha therapy in inflammatory bowel disease: metaanalysis of randomized controlled trials. The American Journal of Gastroenterology. 2013;108(8):1268-1276.

29. Dignass A, Eliakim R, Magro F, et al. Second European evidence-based consensus on the diagnosis and management of ulcerative colitis part 1: definitions and diagnosis. J Crohns Colitis. 2012;6(10):965-990.

30. Jeuring SF, Bours PH, Zeegers MP, et al. Disease outcome of ulcerative colitis in an Era of changing treatment strategies: results from the Dutch population-based IBDSL cohort. Journal of Crohn's \& Colitis. 2015;9(10):837-845.

31. Vester-Andersen MK, Prosberg MV, Jess T, et al. Disease course and surgery rates in inflammatory bowel disease: a population-based, 7-year follow-up study in the era of immunomodulating therapy. The American Journal of Gastroenterology. 2014;109(5):705-714.

32. Kaser A, Zeissig S, Blumberg RS. Inflammatory bowel disease. Annual Review of immunology. 2010;28:573-621.

33. Schulz O, Jaensson E, Persson EK, et al. Intestinal CD103+, but not CX3CR1+, antigen sampling cells migrate in lymph and serve classical dendritic cell functions. The Journal of Experimental Medicine. 2009;206(13):3101-3114. 
34. Mora JR, Bono MR, Manjunath N, et al. Selective imprinting of gut-homing T cells by Peyer's patch dendritic cells. Nature. 2003; 424(6944):88-93.

35. Bekiaris V, Persson EK, Agace WW. Intestinal dendritic cells in the regulation of mucosal immunity. Immunological Reviews. 2014;260(1): 86-101.

36. Schweighoffer T, Tanaka Y, Tidswell M, et al. Selective expression of integrin alpha 4 beta 7 on a subset of human CD4+ memory T cells with Hallmarks of gut-trophism. Journal of Immunology (Baltimore, Md : 1950). 1993;151(2):717-729.

37. Cader MZ, Kaser A. Recent advances in inflammatory bowel disease: mucosal immune cells in intestinal inflammation. Gut. 2013;62(11):1653-1664.

38. McCarthy NE, Bashir Z, Vossenkämper A, et al. Proinflammatory $\mathrm{V} \delta 2+\mathrm{T}$ cells populate the human intestinal Mucosa and enhance IFN- $\gamma$ production by Colonic $\alpha \beta$ T cells. The Journal of Immunology. 2013;191(5):2752-2763.

39. Rott LS, Briskin MJ, Butcher EC. Expression of alpha4beta7 and E-selectin ligand by circulating memory B cells: implications for targeted trafficking to mucosal and systemic sites. Journal of Leukocyte Biology. 2000;68(6):807-814.

40. Berg EL, McEvoy LM, Berlin C, Bargatze RF, Butcher EC. L-selectinmediated lymphocyte rolling on MAdCAM-1. Nature. 1993;366(6456) 695-698.

41. Briskin M, Winsor-Hines D, Shyjan A, et al. Human mucosal addressin cell adhesion molecule-1 is preferentially expressed in intestinal tract and associated lymphoid tissue. The American Journal of Pathology. 1997;151(1):97-110.

42. Berlin C, Berg EL, Briskin MJ, et al. $\alpha 4 \beta 7$ integrin mediates lymphocyte binding to the mucosal vascular addressin MAdCAM-1. Cell. 1993;74(1):185-195.

43. Arihiro S, Ohtani H, Suzuki M, et al. Differential expression of mucosal addressin cell adhesion molecule-1 (MAdCAM-1) in ulcerative colitis and Crohn's disease. Pathology International. 2002;52(5-6):367-374.

44. Holzmann B, Weissman IL. Peyer's patch-specific lymphocyte homing receptors consist of a VLA-4-like alpha chain associated with either of two integrin beta chains, one of which is novel. The EMBO Journal. 1989;8(6):1735-1741.

45. Ghosh S, Goldin E, Gordon FH, et al. Natalizumab for Active Crohn's Disease. New England Journal of Medicine. 2003;348(1):24-32.

46. Chen C-H, Kularatna G, Stone CD, Gutierrez AM, Dassopoulos T. Clinical experience of natalizumab in Crohn's disease patients in a restricted distribution program. Annals of gastroenterology. 2013;26(3):233-238.

47. Fedyk ER, Wyant T, Yang LL, et al. Exclusive antagonism of the alpha4 beta7 integrin by vedolizumab confirms the gut-selectivity of this pathway in primates. Inflammatory Bowel Diseases. 2012;18(11):2107-2119.

48. Trivedi PJ, Bruns T, Ward S, et al. Intestinal CCL25 expression is increased in colitis and correlates with inflammatory activity. J Autoimmun. 2016;68:98-104.

49. Wendt E, Keshav S. CCR9 antagonism: potential in the treatment of Inflammatory Bowel Disease. Clinical and Experimental Gastroenterology. 2015;8:119-130.

50. Nguyen LP, Pan J, Dinh TT, et al. Role and species-specific expression of colon T cell homing receptor GPR15 in colitis. Nat Immunol. 2015;16(2):207-213.

51. Byrareddy SN, Arthos J, Cicala C, et al. Sustained virologic control in SIV + macaques after antiretroviral and $\alpha 4 \beta 7$ antibody therapy. Science. 2016;354(6309):197-202.

52. Feagan BG, Greenberg GR, Wild G, et al. Treatment of Ulcerative Colitis with a Humanized Antibody to the $\alpha 4 \beta 7$ Integrin. New England Journal of Medicine. 2005;352(24):2499-2507.

53. Rosario M, Dirks NL, Gastonguay MR, et al. Population pharmacokinetics-pharmacodynamics of vedolizumab in patients with ulcerative colitis and Crohn's disease. Alimentary Pharmacology \& Therapeutics 2015;42(2):188-202.

54. Fasanmade AA, Adedokun OJ, Ford J, et al. Population pharmacokinetic analysis of infliximab in patients with ulcerative colitis. Eur J Clin Pharmacol. 2009;65(12):1211-1228.
55. Parikh A, Leach T, Wyant T, et al. Vedolizumab for the treatment of active ulcerative colitis: a randomized controlled phase 2 dose-ranging study. Inflammatory Bowel Diseases. 2012;18(8):1470-1479.

56. Feagan BG, Rutgeerts P, Sands BE, et al. Vedolizumab as induction and maintenance therapy for ulcerative colitis. New England journal of Medicine. 2013;369(8):699-710.

57. Sandborn WJ, Feagan BG, Rutgeerts P, et al. Vedolizumab as induction and maintenance therapy for Crohn's disease. New England journal of Medicine. 2013;369(8):711-721.

58. Colombel JF, Sandborn WJ, Reinisch W, et al. Infliximab, Azathioprine, or Combination Therapy for Crohn's Disease. New England Journal of Medicine. 2010;362(15):1383-1395.

59. Maini RN, Breedveld FC, Kalden JR, et al. Therapeutic efficacy of multiple intravenous infusions of anti-tumor necrosis factor alpha monoclonal antibody combined with low-dose weekly methotrexate in rheumatoid arthritis. Arthritis and Rheumatism. 1998;41(9):1552-1563.

60. Earp J, Fang L, Lian M, et al. Assessing labeling claims for drug interactions using a population PK approach: Vedolizumab. Journal of Pharmacokinetics and Pharmacodynamics. 2014;41(1):S39.

61. Neurath MF, Travis SP. Mucosal healing in inflammatory bowel diseases: a systematic review. Gut. 2012;61(11):1619-1635.

62. Pineton de Chambrun G, Peyrin-Biroulet L, Lemann M, Colombel JF. Clinical implications of mucosal healing for the management of IBD. Nature Reviews Gastroenterology \& Hepatology. 2010;7(1):15-29.

63. Feagan BG, McDonald J, Greenberg G, et al. An ascending dose trial of humanized A4B7 antibody in ulcerative colitis. Gastroenterology. 2000;118 (4 Suppl 2):A874.

64. Mosli MH, MacDonald JK, Bickston SJ, et al. Vedolizumab for induction and maintenance of remission in ulcerative colitis: a Cochrane systematic review and meta-analysis. Inflammatory Bowel Diseases. 2015;21(5):1151-1159.

65. Salleron J, Danese S, D’Agay L, Peyrin-Biroulet L. Effectiveness Research in Inflammatory Bowel Disease: A Necessity and a Methodological Challenge. Journal of Crohn's \& Colitis. 2016;10(9):1096-1102.

66. Keane J, Gershon S, Wise RP, et al. Tuberculosis associated with infliximab, a tumor necrosis factor alpha-neutralizing agent. The New England Journal of Medicine. 2001;345(15):1098-1104.

67. Christensen B, Goeppinger SR, Colman R, et al. Post-marketing experience of vedolizumab for IBD: The University of Chicago experience. Journal of Crohn's \& Colitis. 2015;9(Suppl 1):S388-S389.

68. Walmsley RS, Ayres RC, Pounder RE, Allan RN. A simple clinical colitis activity index. Gut. 1998;43(1):29-32.

69. Shelton E, Allegretti JR, Stevens B, et al. Efficacy of Vedolizumab as Induction Therapy in Refractory IBD Patients: A Multicenter Cohort. Inflammatory Bowel Diseases. 2015;21(12):2879-2885.

70. Vivio EE, Kanuri N, Gilbertsen JJ, et al. Vedolizumab Effectiveness and Safety Over the First Year of Use in an IBD Clinical Practice. Journal of Crohn's \& Colitis. 2016;10(4):402-409.

71. Amiot A, Grimaud JC, Peyrin-Biroulet L, et al. Effectiveness and Safety of Vedolizumab Induction Therapy for Patients With Inflammatory Bowel Disease. Clinical Gastroenterology and Hepatology. 2016;14(11):1593-1601.

72. Baumgart DC, Bokemeyer B, Drabik A, Stallmach A, Schreiber S, Vedolizumab Germany C. Vedolizumab induction therapy for inflammatory bowel disease in clinical practice - a nationwide consecutive German cohort study. Alimentary Pharmacology \& Therapeutics. 2016;43(10):1090-1102.

73. Stallmach A, Langbein C, Atreya R, et al. Vedolizumab provides clinical benefit over 1 year in patients with active inflammatory bowel disease - a prospective multicenter observational study. Alimentary pharmacology \& therapeutics. 2016;44(11-12):1199-1212.

74. Samaan MA, Pavlidis P, Johnston E, et al. Vedolizumab: early experience and medium-term outcomes from two UK tertiary IBD centres. Frontline Gastroenterology. 2016 Published Online First: [10 Aug 2016] doi:10.1136/flgastro-2016-100720

75. Lin L, Liu X, Wang D, Zheng C. Efficacy and safety of antiintegrin antibody for inflammatory bowel disease: a systematic review and meta-analysis. Medicine. 2015;94(10):e556. 
76. Danese S, Fiorino G, Peyrin-Biroulet L, et al. Biological agents for moderately to severely active ulcerative colitis: a systematic review and network meta-analysis. Annals of Internal Medicine. 2014;160(10): 704-711.

77. Grant AJ, Lalor PF, Hubscher SG, Briskin M, Adams DH. MAdCAM-1 expressed in chronic inflammatory liver disease supports mucosal lymphocyte adhesion to hepatic endothelium (MAdCAM-1 in chronic inflammatory liver disease). Hepatology. 2001;33(5): 1065-1072.

78. Eksteen B, Heatherington J, Oshiomogho JI, Panaccione R, Kaplan G, Ghosh S. 812f efficacy and safety of induction dosing of Vedolizumab for reducing biliary inflammation in Primary Sclerosing Cholangitis (PSC) in individuals with inflammatory bowel disease. Gastroenterology.150(4):S1268.

79. Lim TY, Pavlidis P, Pirani T, et al. Vedolizumab in inflammatory bowel disease associated with Autoimmune Liver Disease pre- and post-liver transplantation: a case series. Inflammatory Bowel Diseases. 2016;22(10):E39-E40.

80. Bloomgren G, Richman S, Hotermans C, et al. Risk of natalizumabassociated progressive multifocal leukoencephalopathy. New England Journal of Medicine. 2012;366(20):1870-1880.

81. Haanstra KG, Hofman SO, Lopes Estevao DM, et al. Antagonizing the alpha4beta1 integrin, but not alpha4beta7, inhibits leukocytic infiltration of the central nervous system in rhesus monkey experimental autoimmune encephalomyelitis. Journal of immunology. 2013;190(5): 1961-1973.

82. Stuve O, Marra CM, Jerome KR, et al. Immune surveillance in multiple sclerosis patients treated with natalizumab. Annals of neurology. 2006;59(5):743-747.
83. Wyant T, Leach T, Sankoh S, et al. Vedolizumab affects antibody responses to immunisation selectively in the gastrointestinal tract: randomised controlled trial results. Gut. 2015;64(1):77-83.

84. Colombel JF, Sands BE, Rutgeerts P, et al. The safety of vedolizumab for ulcerative colitis and Crohn's disease. Gut. 2016; Online First: [18 Feb 2016] doi:10.1136/gutjnl-2015-311079

85. Irvine EJ, Feagan B, Rochon J, et al. Quality of life: a valid and reliable measure of therapeutic efficacy in the treatment of inflammatory bowel disease. Canadian Crohn's Relapse Prevention Trial Study Group. Gastroenterology. 1994;106(2):287-296.

86. Feagan BG, Patel H, Colombel JF, et al. Effects of vedolizumab on health-related quality of life in patients with ulcerative colitis: results from the randomised GEMINI 1 trial. Alimentary pharmacology \& therapeutics. 2017;45(2):264-275.

87. Kane S, Dixon L. Adherence rates with infliximab therapy in Crohn's disease. Alimentary Pharmacology \& Therapeutics. 2006;24(7): 1099-1103.

88. van der Have M, Oldenburg B, Kaptein AA, et al. Non-adherence to anti-TNF therapy is associated with illness perceptions and clinical outcomes in outpatients with inflammatory bowel disease: results from a prospective multicentre study. Journal of Crohn's \& Colitis. 2016;10(5): 549-555.

89. Tew GW, Hackney JA, Gibbons D, et al. Association between response to Etrolizumab and expression of integrin alpha E and Granzyme A in colon biopsies of patients with ulcerative colitis. Gastroenterology. 2016; 150(2):477-487.

90. Duncan J, Caulfield S, Clark A, Anderson S, Sanderson J, Irving P. PTH-071 A multidisciplinary virtual biologics clinic: is it worthwhile? Gut. 2015;59(Suppl 1):A151-A152.
Clinical and Experimental Gastroenterology

\section{Publish your work in this journal}

Clinical and Experimental Gastroenterology is an international, peerreviewed, open access, online journal publishing original research, reports, editorials, reviews and commentaries on all aspects of gastroenterology in the clinic and laboratory. This journal is included on PubMed. The manuscript management system is completely online

\section{Dovepress}

and includes a very quick and fair peer-review system, which is all easy to use. Visit http://www.dovepress.com/testimonials.php to read real quotes from published authors. 